\title{
Quasiparticle Approach to Molecules Interacting with Quantum Solvents
}

\author{
Mikhail Lemeshko* \\ IST Austria (Institute of Science and Technology Austria), Am Campus 1, 3400 Klosterneuburg, Austria \\ and Kavli Institute for Theoretical Physics, University of California, \\ Santa Barbara, California 93106, USA \\ (Received 13 October 2016; published 27 February 2017)
}

\begin{abstract}
Understanding the behavior of molecules interacting with superfluid helium represents a formidable challenge and, in general, requires approaches relying on large-scale numerical simulations. Here, we demonstrate that experimental data collected over the last 20 years provide evidence that molecules immersed in superfluid helium form recently predicted angulon quasiparticles [Phys. Rev. Lett. 114, 203001 (2015)]. Most important, casting the many-body problem in terms of angulons amounts to a drastic simplification and yields effective molecular moments of inertia as straightforward analytic solutions of a simple microscopic Hamiltonian. The outcome of the angulon theory is in good agreement with experiment for a broad range of molecular impurities, from heavy to medium-mass to light species. These results pave the way to understanding molecular rotation in liquid and crystalline phases in terms of the angulon quasiparticle.
\end{abstract}

DOI: 10.1103/PhysRevLett.118.095301

Among its many peculiar properties, superfluid ${ }^{4} \mathrm{He}$ is quite averse to mixing with impurities which could serve as a microscopic probe of the superfluid phase. As a result, for several decades after the discovery of superfluidity by Allen, Misener, and Kapitza [1,2], only macroscopichydrodynamic_-properties of superfluid helium have been studied in the laboratory. In the 1990s, however, it was demonstrated that atoms and molecules can be trapped in superfluid helium if the latter forms little droplets containing on the order of 1000 helium atoms [3-7]. Over the following years, trapping atoms, molecules, and ions inside superfluid helium nanodropletssometimes called "nanocryostats"-emerged as an important tool of molecular spectroscopy [6-12]. Such nanodroplets allow us to trap single molecules in a cold environment ( 0.4 Kelvin), thereby isolating them from external perturbations. This allows us to record spectra free of collisional and Doppler broadening, as well as to study species that are unstable in the gas phase, such as free radicals.

While superfluid helium does not cause a substantial broadening of molecular spectral lines, it affects molecular rotation. In particular, molecules in superfluid helium nanodroplets acquire an effective moment of inertia that is larger compared to its gas-phase value [6,9]. The relative magnitude of the effect increases from lighter to heavier species and is somewhat similar to renormalization of the effective mass for electrons interacting with a crystalline lattice [13-16].

Semiclassically, molecular rotation in helium can be rationalized within the "adiabatic following" model $[6,7,17-22]$. There, it is assumed that the molecule induces a local density deformation (a "nonsuperfluid shell") of helium which corotates along with the molecule, thereby increasing its moment of inertia. However, such a classical approach does not allow us to get insight into the intriguing aspects of the problem arising from quantum many-body physics. Helium, on the other hand, represents a dense, strongly interacting superfluid, where only a tiny fraction of $6 \%-8 \%$ forms a Bose-Einstein condensate, even at zero temperature [23]. As a result, a detailed quantum mechanical understanding of molecular impurities in helium requires first-principles approaches based on extensive numerical simulations [7]. During the last years, several numerical studies, based mainly on path-integral, variational, and diffusion quantum Monte Carlo (MC) calculations, have been performed for molecules embedded in finite-size $\mathrm{He}_{n}$ clusters with $n \lesssim 100$ [22,24-46].

In this Letter, we show that such an involved manyparticle problem simplifies tremendously, if one assumes that molecules in helium droplets form angulons-recently introduced quasiparticles consisting of a quantum rotor dressed by a field of many-body excitations [47-54]. The angulon theory is inherently many body and describes interactions between a molecule and an infinite number of helium atoms. Nevertheless, it still allows us to derive the effective molecular rotational constants as simple analytic solutions of a microscopic Hamiltonian and assign them a transparent physical interpretation. Moreover, the resulting agreement of the angulon theory with experiment provides strong evidence for the angulon formation inside helium droplets.

We start from introducing the angulon Hamiltonian, which describes interactions of a rotating molecule with a bosonic bath [47]: 


$$
\begin{aligned}
\hat{H}= & B \hat{\mathbf{J}}^{2}+\sum_{k \lambda \mu} \omega_{k} \hat{b}_{k \lambda \mu}^{\dagger} \hat{b}_{k \lambda \mu} \\
& +\sum_{k \lambda \mu} \sqrt{\frac{4 \pi}{2 \lambda+1}} U_{\lambda}(k)\left[Y_{\lambda \mu}^{*}(\hat{\theta}, \hat{\phi}) \hat{b}_{k \lambda \mu}^{\dagger}+Y_{\lambda \mu}(\hat{\theta}, \hat{\phi}) \hat{b}_{k \lambda \mu}\right],
\end{aligned}
$$

where $Y_{\lambda \mu}(\hat{\theta}, \hat{\phi})$ are spherical harmonics [55], $\sum_{k} \equiv \int d k$, and $\hbar \equiv 1$. The first term of Eq. (1) corresponds to the rotational kinetic energy of the molecule, with $\hat{\mathbf{J}}$ the angular momentum operator. $B=1 /(2 I)$ is the molecular rotational constant, where $I$ is the molecular moment of inertia. While the first term of Eq. (1) describes rotations of a linear rigid rotor, one can use it to describe an average kinetic energy of more complex molecules, such as symmetric and asymmetric tops [56,57], aiming to obtain an average renormalization of their rotational constants. Thus, the bare eigenstates of the impurity are given by the $(2 L+1)$-fold degenerate levels $|L, M\rangle$ with energies $E_{L}=B L(L+1)$, where $L$ is the angular momentum quantum number and $M$ its projection on the laboratoryframe $z$ axis. The second term of the Hamiltonian (1) represents the kinetic energy of the superfluid excitations (such as phonons and rotons), as given by the dispersion relation $\omega_{k}$. Here, the operators $\hat{b}_{k \lambda \mu}^{\dagger}\left(\hat{b}_{k \lambda \mu}\right)$ are creating (annihilating) a bath excitation with linear momentum $k=|\mathbf{k}|$, the angular momentum $\lambda$, and its projection $\mu$, onto the $z$ axis. These operators can be obtained from the spherical-harmonic expansion of the usual creation and annihilation operators, $\hat{b}_{\mathbf{k}}^{\dagger}$ and $\hat{b}_{\mathbf{k}}$, defined in Cartesian space; see Refs. [47-49] for details.

The last term of the angulon Hamiltonian (1) describes the interaction between the molecular impurity and the superfluid, where the coupling constants $U_{\lambda}(k)$ are proportional to the Legendre moments of the molecule-helium potential energy surface (PES) in Fourier space. Note that the impurity-bath coupling explicitly depends on molecular angle operators, $(\hat{\theta}, \hat{\phi})$, which makes Eq. (1) substantially different from, e.g., the Bose-polaron [16] or the spinboson [58] models. The Hamiltonian (1) was originally derived to describe an ultracold molecule interacting with a dilute Bose-Einstein condensate, where the coupling constants $U_{\lambda}(k)$ assume a simple analytic form [47,49]. In order to reproduce experimental data for a dense superfluid of ${ }^{4} \mathrm{He}$, however, we will approach Eq. (1) from a phenomenological perspective, by analogy with effective field theories of nuclear [59] and condensed matter [60] physics.

Namely, we use a simple, one-parameter model to extract $U_{\lambda}(k)$ from the $a b$ initio PES calculations available in the literature. First, we note that broadening of the spectral lines in superfluid helium [61] and solid para- $\mathrm{H}_{2}$ [62] is dominated by rotational dephasing as opposed to decay. Therefore, we can assume that $U_{\lambda}$ 's with even $\lambda$ play the main role, since they can lead to boson scattering which preserves molecular angular momentum. Furthermore, since for most molecules, the $\lambda=2$ channel is dominant [63], for the sake of simplicity we neglect the rest of the $U_{\lambda}$ terms.

Second, we assume that the coupling constant in the $\lambda=2$ channel can be approximated as

$$
U_{2}(k)=\Delta f(k) .
$$

Here, the form factor $f(k)$ is considered to be the same for all the molecular species, while the anisotropy parameter $\Delta$ depends on a particular molecule. Thus, the strength of the molecule-superfluid interactions can be quantified by the dimensionless parameter

$$
\gamma=B / \Delta
$$

and the species with $\gamma<1$ and $\gamma>1$ belong to the strongcoupling and weak-coupling regimes, respectively.

We evaluate the anisotropy parameter $\Delta$ as

$$
\Delta=\frac{\left|V_{\mathrm{eff}}^{\|}-V_{\mathrm{eff}}^{\perp}\right|}{2} \sqrt{\frac{5}{4 \pi}},
$$

where $V_{\text {eff }}^{\|}$and $V_{\text {eff }}^{\perp}$ are the effective molecule-helium interactions, derived from the $a b$ initio PES calculations [24,64-84] as follows [85]. For linear molecules, $V_{\mathrm{eff}}^{\|}$and $V_{\text {eff }}^{\perp}$ correspond to the effective molecule-helium interactions in the linear and $T$-shaped geometries, respectively. In most cases, the values of $V_{\text {eff }}$ were set to the average depths of the minima or saddle points in the corresponding configurations. If for one of the configurations the PES was purely repulsive, the corresponding $V_{\text {eff }}$ was set to 0 , to reflect the fact that the helium density vanishes in this region. For symmetric and asymmetric tops $\left(\mathrm{CH}_{3}\right.$, $\mathrm{NH}_{3}, \mathrm{H}_{2} \mathrm{O}$ ), $V_{\text {eff }}^{\|}$was evaluated along the main molecular symmetry axis, while $V_{\text {eff }}^{\perp}$ along the direction perpendicular to it, laying within the mirror symmetry plane of the system. For the spherical-top molecules $\left(\mathrm{SF}_{6}, \mathrm{CH}_{4}\right), \Delta$ was evaluated as an average anisotropy of PES minima, which are not symmetry equivalent. In this case, $\left|V_{\text {eff }}^{\|}-V_{\text {eff }}^{\perp}\right|$ in Eq. (4) was replaced by $\sum_{i \neq j}\left|V_{\text {eff }}^{(i)}-V_{\text {eff }}^{(j)}\right|$, where $i, j$ label all nonequivalent minima of the PES [85].

We would like to emphasise that we are quite aware of the fact that such a one-parameter model provides a very rough approximation to the two-body interaction potential. However, as we can see below, it suffices to obtain a good agreement with experiment.

For molecules in helium droplets, the low-energy rotational spectrum is usually approximated as $E_{L} \approx$ $B^{*} L(L+1)$, where $B^{*}$ is the effective rotational constant [6]. Let us first derive $B^{*}$ from Eq. (1) in the strongly interacting regime $\gamma \ll 1$. Getting insight into this regime 
is inherently challenging, since it involves coupling molecular rotational angular momentum to angular momenta of, in principle, an infinite number of superfluid excitations. However, the solution can be drastically simplified by using a canonical transformation recently introduced by Schmidt and Lemeshko [48]:

$$
\hat{S}=e^{-i \hat{\phi} \otimes \hat{\Lambda}_{z}} e^{-i \hat{\theta} \otimes \hat{\Lambda}_{y}} e^{-i \hat{\gamma} \otimes \hat{\Lambda}_{z}} .
$$

Here, $(\hat{\phi}, \hat{\theta}, \hat{\gamma})$ are the angle operators which act in the Hilbert space of the molecular rotor, and

$$
\hat{\boldsymbol{\Lambda}}=\sum_{k \lambda \mu \nu} \hat{b}_{k \lambda \mu}^{\dagger} \boldsymbol{\sigma}_{\mu \nu}^{\lambda} \hat{b}_{k \lambda \nu}
$$

is the total angular momentum operator of the superfluid excitations, acting in their corresponding Hilbert space. The matrices $\boldsymbol{\sigma}^{\lambda} \equiv\left\{\sigma_{-1}^{\lambda}, \sigma_{0}^{\lambda}, \sigma_{+1}^{\lambda}\right\}$ fulfill the $S O(3)$ algebra in the representation of angular momentum $\lambda$. Thus, the transformation operator of Eq. (5) transfers the superfluid degrees of freedom into the frame corotating along with the molecule.

The transformation (5) brings the Hamiltonian (1) to the following form [48]:

$$
\begin{aligned}
\hat{\mathcal{H}} \equiv & \hat{S}^{-1} \hat{H} \hat{S}=B(\hat{\mathbf{L}}-\hat{\boldsymbol{\Lambda}})^{2}+\sum_{k \lambda \mu} \omega_{k} \hat{b}_{k \lambda \mu}^{\dagger} \hat{b}_{k \lambda \mu} \\
& +\sum_{k \lambda} U_{\lambda}(k)\left[\hat{b}_{k \lambda 0}^{\dagger}+\hat{b}_{k \lambda 0}\right],
\end{aligned}
$$

where $\hat{\mathbf{L}}=\hat{\mathbf{J}}+\hat{\mathbf{\Lambda}}$ is the total angular momentum of the system, which acts in the rotating frame of the impurity and therefore obeys anomalous commutation relations $[48,86,87]$.

In the limit of $\gamma \rightarrow 0$, the transformed Hamiltonian (7) can be diagonalized exactly, with the ground state for each $|L M\rangle$ given by

$$
\left|\psi_{L M}\right\rangle=e^{\sum_{k \lambda}\left[U_{\lambda}(k) / \omega_{k}\right]\left(\hat{b}_{k \lambda 0}-\hat{b}_{k \lambda 0}^{\dagger}\right)}|0\rangle|L M\rangle .
$$

Equations (7) and (8) provide a transparent physical interpretation of molecular interactions with a superfluid. For a slowly rotating molecule, the superfluid coherent state of Eq. (8) does not change upon molecular rotation. In a way, it can be thought of as a quantum formulation of the "nonsuperfluid helium shell" which rotates along with the molecule $[6,7,17]$. On the other hand, the effective molecular angular momentum, cf. the first term of Eq. (7), is given by the difference between the total angular momentum of the system $\hat{\mathbf{L}}$ and the superfluid angular momentum $\hat{\mathbf{\Lambda}}$. Thus, the energy of a state with a given total angular momentum $L$ is lower in the presence of a superfluid $(\hat{\boldsymbol{\Lambda}} \neq 0)$ compared to a free molecule $(\hat{\boldsymbol{\Lambda}}=0)$, which leads to an effective renormalization of the rotational constant.

In the strong-coupling limit, the angular momentum of the superfluid is given by

$$
\left\langle\hat{\boldsymbol{\Lambda}}^{2}\right\rangle \equiv\left\langle\psi_{L M}\left|\hat{\boldsymbol{\Lambda}}^{2}\right| \psi_{L M}\right\rangle=\sum_{k \lambda} \lambda(\lambda+1) \frac{U_{\lambda}^{2}(k)}{\omega_{k}^{2}} .
$$

In the first order, we can assume that $\hat{\mathbf{\Lambda}} \uparrow \uparrow \hat{\mathbf{L}}$, i.e., $\hat{\mathbf{\Lambda}}=\alpha(L) \hat{\mathbf{L}}$, where the proportionality constant can be calculated as $\alpha^{2}(L)=\left\langle\hat{\boldsymbol{\Lambda}}^{2}\right\rangle / L(L+1)$, with $\left\langle\hat{\boldsymbol{\Lambda}}^{2}\right\rangle$ given by Eq. (9). Since in experiment the value of $B^{*}$ is usually determined from the splitting between the two lowest rotational states [6], we evaluate it from the first term of Eq. (7) for $L=1$ :

$$
\frac{B_{\mathrm{SC}}^{*}}{B}=(1-\eta \Delta)^{2},
$$

where $\eta \equiv \alpha(1) / \Delta=\left(3 \sum_{k} f^{2}(k) / \omega_{k}^{2}\right)^{1 / 2}$ is the only phenomenological parameter of the strong-coupling theory, which is obtained by fitting to the experimental data [85].

Figures 1(a) and 1(b) show the comparison of Eq. (10) (red circles) with experiment (empty squares). We see that a good agreement with experiment is achieved for most molecules with $\gamma<1$ : $\mathrm{SF}_{6}, \mathrm{CS}_{2}, \mathrm{HCCCN}, \mathrm{OCS}, \mathrm{N}_{2} \mathrm{O}$, $\mathrm{CO}_{2}, \mathrm{CO}$, and NO. For $\mathrm{I}_{2}$, the model overestimates the value of $B^{*} / B$ compared to the MC result $[54,88]$ by about $20 \%$. It is worth noting that the calculations of impulsive alignment for $I_{2}$ in helium droplets performed with the MC value of $B^{*} / B=0.6$ predict the first revival later than observed in experiment [54], which signals that the experimental value of $B^{*} / B$ might be larger than 0.6.

For $\mathrm{C}_{2} \mathrm{H}_{2}, \mathrm{DCN}$, and $\mathrm{HCN}$ - see Fig. 1(b) - the disagreement with experiment is substantially larger. This fact might be due to the interplay between phonon and roton excitations in this region of rotational constants [34,35,43], which can potentially lead to a nontrivial dependence of the parameter $\eta$ on $B$ and $\Delta$. We note that for these three species, classical hydrodynamics calculations lead to an underestimated $B^{*} / B$ ratio as well $[18,19]$. For $\mathrm{LiH}$ [Fig. 1(b)], strong renormalization of the rotational constant was previously predicted using path-integral MC simulations [44]. Here, due to a pronounced anisotropy of the He-LiH PES [75], the strong-coupling angulon theory predicts $\langle\hat{\boldsymbol{\Lambda}}\rangle>L$, which indicates the breakdown of the employed approximations. We attribute it to the fact that the PES features strong $U_{1}(k)$ components which lead to processes involving simultaneous absorption or emission of two phonons with $\lambda=1$, preserving the molecular angular momentum. The latter are not accounted for by the present theory. Nevertheless, Eq. (10) predicts a strong decrease of the $\mathrm{LiH}$ rotational constant in helium, which is in fair agreement with predictions of Ref. [44]. For light molecules of Fig. 1(c), the results of the strong-coupling theory substantially deviate from experiment and are therefore not shown.

Now, let us consider the regime of weak coupling $\gamma>1$. There, one can obtain the energies of molecular states in helium using second-order perturbation theory on the Hamiltonian (1): 

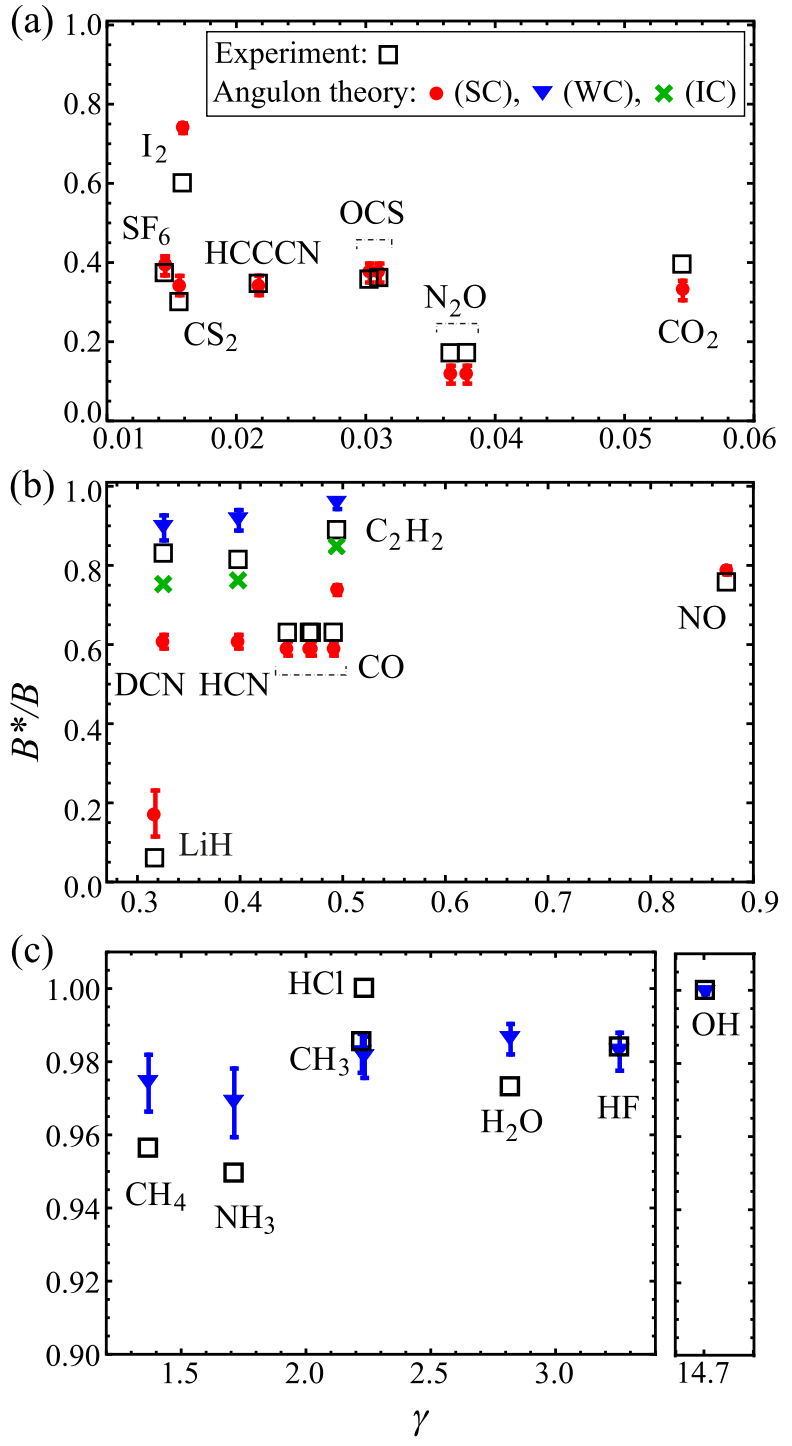

FIG. 1. Renormalization of the molecular rotational constant $B^{*} / B$ as a function of the coupling parameter $\gamma$. The panels correspond to (a) heavy molecules, (b) medium-mass molecules, and (c) light molecules. Experimental data from Refs. [17,26,34,35,43,44,54,88-101] (empty squares) are compared with the angulon theory in the strong-coupling regime, Eq. (10) with $\eta=[0.060 \pm 0.003] / \mathrm{cm}^{-1}$ (red circles), and the weak-coupling regime, Eq. (12) with $\xi=[0.0092 \pm 0.0028] / \mathrm{cm}^{-1}$ (blue triangles). Green crosses show the intermediate-coupling interpolation between the strong- and weak-coupling theories.

$E_{L}=B L(L+1)-\sum_{k \lambda L^{\prime}} \frac{U_{\lambda}(k)^{2}\left[C_{L 0, \lambda 0}^{L^{\prime} 0}\right]^{2}}{B L^{\prime}\left(L^{\prime}+1\right)-B L(L+1)+\omega_{k}}$,

where $C_{L 0, \lambda 0}^{L^{\prime} 0}$ is the Clebsch-Gordan coefficient [55]. The second term of Eq. (11) provides a shift which depends on the molecular rotational state $L$ and therefore leads to renormalization of the rotational constant. It is interesting to note that the process described by Eq. (11)—differential shifts of the molecular rotational levels due to virtual phonon absorption-represents an exact analogue of the Lamb shift in quantum electrodynamics, which is induced by virtual photon excitations $[102,103]$.

Taking into account the dominant processes with $L=L^{\prime}$ in Eq. (11), we obtain

$$
\frac{B_{\mathrm{WC}}^{*}}{B}=1-\xi \frac{\Delta^{2}}{B},
$$

where $\xi=\sum_{k} f^{2}(k) /\left(5 \omega_{k}\right)$ is the only free parameter of the weak-coupling theory, obtained by fitting to the experimental data [85].

Figure 1(c) compares Eq. (12) (blue triangles) with experimental data for light molecules. One can see that an agreement within $2 \%$ is achieved for all the considered species: $\mathrm{CH}_{4}, \mathrm{CH}_{3}, \mathrm{NH}_{3}, \mathrm{HCl}, \mathrm{H}_{2} \mathrm{O}, \mathrm{OH}$, and $\mathrm{HF}$, which indicates the applicability of the weak-coupling angulon theory. We attribute a slightly larger disagreement for $\mathrm{CH}_{4}$, $\mathrm{NH}_{3}$, and $\mathrm{H}_{2} \mathrm{O}$ to the approximation to the PES [Eq. (2)]. We would like to point out that since the experiments on $\mathrm{HCl}$ [94] and $\mathrm{OH}$ [96] did not detect any significant renormalization of the rotational constant, the corresponding experimental values of $B^{*} / B$ were set to 1 . While our theory indeed predicts $B^{*} / B \approx 1$ for the case of $\mathrm{OH}$, we observe $B^{*} / B \approx$ 0.98 for $\mathrm{HCl}$, which is quite close to the corresponding value for HF. Given the similarities between the two species, we hope that this result will stimulate further measurements of $B^{*} / B$ for $\mathrm{HCl}$. For most heavy and medium-mass species [Figs. 1(a) and 1(b)], the weak-coupling theory fails to reproduce experimental data and is therefore not presented.

A peculiar situation occurs for three of the studied molecules, namely, $\mathrm{C}_{2} \mathrm{H}_{2}, \mathrm{DCN}$, and $\mathrm{HCN}$. There, the weak-coupling theory overestimates the $B^{*} / B$ ratio, while the strong-coupling approach underestimates it. In principle, in order to obtain a quantitative agreement with experiment for these particular species, a different, intermediatecoupling angulon theory is required. However, as a rough approximation we can estimate the intermediate-coupling results by interpolating between the weak-coupling and strong-coupling theories as $B_{\mathrm{IC}}^{*}=\left(B_{\mathrm{SC}}^{*}+B_{\mathrm{WC}}^{*}\right) / 2$. The values of $B_{\mathrm{IC}}^{*} / B$ are shown in Fig. 1(b) by green crosses and are seen to provide a good agreement with experiment.

In addition to $B$ renormalization, previous experiments reported a significant increase in the centrifugal distortion constants $D$, compared to the gas phase, obtaining, e.g., $D=3.7 \times 10^{-5} \mathrm{~cm}^{-1}$ for $\mathrm{SF}_{6}[100], D=1.6 \times 10^{-4} \mathrm{~cm}^{-1}$ for HCCCN [71], and $D=3.8 \times 10^{-4} \mathrm{~cm}^{-1}$ for OCS [17]. Such a distortion comes from the coupling between the angular momenta $\hat{\mathbf{L}}$ and $\hat{\mathbf{\Lambda}}$ in Eq. (7) [20]. In order to obtain accurate values for $D$, a more involved, all-coupling angulon theory is required. Here, we perform a rough estimate, based on second-order perturbation theory, which gives $D \sim \gamma^{2} /(5 \xi)$ [85]. For the molecules mentioned above, we obtain $D \sim 4 \times 10^{-3}, 1 \times 10^{-2}$, and $2 \times 10^{-2} \mathrm{~cm}^{-1}$, respectively. Although this estimate significantly exceeds the measured values, the drastic increase of $D$ in helium, as 
well as its qualitative change from molecule to molecule, are in agreement with experiment.

In summary, we have demonstrated that the angulon theory is able to reproduce experimental data on the renormalization of rotational constants in superfluid ${ }^{4} \mathrm{He}$ for 25 different molecules, based on only two phenomenological parameters. It has been shown that in the strongcoupling regime (mostly taking place for heavy and medium-mass molecules), the renormalization of molecular moments of inertia occurs due to a macroscopic deformation of the superfluid, which leads to redistribution of angular momentum between the molecule and excitations in helium. In the weak-coupling regime (applicable to lighter species), the change in $B$ takes place due to a rotational Lamb shift induced by virtual single-phonon excitations.

These results provide strong evidence that molecules immersed in superfluid ${ }^{4} \mathrm{He}$ indeed form the angulon quasiparticles and open the door for substantial simplifications of existing theories. As an example, the angulon theory is straightforward to apply to large polyatomic molecules and complexes studied in experiment $[6,7,9]$ and can be extended to time-dependent problems of molecular dynamics in ${ }^{4} \mathrm{He}$ [54,104-106]. Moreover, the applicability of the angulon theory is not limited to bosonic quantum liquids. Therefore, it can potentially serve as a building block to understand molecular rotation in other types of solutions and solid-state environments.

We thank Gary Douberly and Bretislav Friedrich for insightful discussions and Robert Zillich for sharing unpublished numerical results [88]. This research was supported in part by the National Science Foundation under Grant No. NSF PHY11-25915.

*mikhail.lemeshko@ist.ac.at

[1] J. F. Allen and A. D. Misener, Nature (London) 141, 75 (1938).

[2] P. Kapitza, Nature (London) 141, 74 (1938).

[3] S. Goyal, D. L. Schutt, and G. Scoles, Phys. Rev. Lett. 69, 933 (1992).

[4] M. Hartmann, R. E. Miller, J. P. Toennies, and A. Vilesov, Phys. Rev. Lett. 75, 1566 (1995).

[5] S. Grebenev, J. P. Toennies, and A. F. Vilesov, Science 279, 2083 (1998)

[6] J. P. Toennies and A. F. Vilesov, Angew. Chem., Int. Ed. Engl. 43, 2622 (2004).

[7] K. Szalewicz, Int. Rev. Phys. Chem. 27, 273 (2008).

[8] J. P. Toennies and A. F. Vilesov, Annu. Rev. Phys. Chem. 49, 1 (1998).

[9] C. Callegari, K. K. Lehmann, R. Schmied, and G. Scoles, J. Chem. Phys. 115, 10090 (2001).

[10] M. Y. Choi, G. E. Douberly, T. M. Falconer, W. K. Lewis, C. M. Lindsay, J. M. Merritt, P. L. Stiles, and R. E. Miller, Int. Rev. Phys. Chem. 25, 15 (2006).

[11] F. Stienkemeier and K. K. Lehmann, J. Phys. B 39, R127 (2006).
[12] M. Mudrich and F. Stienkemeier, Int. Rev. Phys. Chem. 33, 301 (2014).

[13] J. Appel, in Solid State Physics, Vol. 21, edited by H. Ehrenreich, F. Seitz, and D. Turnbull (Academic, New York, 1968).

[14] D. Emin, Polarons (Cambridge University Press, Cambridge, England, 2013).

[15] Polarons and Excitons, edited by C. Kuper and G. D. Whitfield (Plenum, New York, 1962).

[16] J. T. Devreese, arXiv:1012.4576.

[17] S. Grebenev, M. Hartmann, M. Havenith, B. Sartakov, J. P. Toennies, and A. F. Vilesov, J. Chem. Phys. 112, 4485 (2000).

[18] C. Callegari, A. Conjusteau, I. Reinhard, K. K. Lehmann, G. Scoles, and F. Dalfovo, Phys. Rev. Lett. 83, 5058 (1999).

[19] C. Callegari, A. Conjusteau, I. Reinhard, K. K. Lehmann, G. Scoles, and F. Dalfovo, Phys. Rev. Lett. 84, 1848 (2000).

[20] K. K. Lehmann, J. Chem. Phys. 114, 4643 (2001).

[21] K. K. Lehmann, J. Chem. Phys. 117, 1595 (2002).

[22] M. V. Patel, A. Viel, F. Paesani, P. Huang, and K. B. Whaley, J. Chem. Phys. 118, 5011 (2003).

[23] A. J. Leggett, Quantum Liquids: Bose Condensation and Cooper Pairing in Condensed-Matter Systems (Oxford University Press, New York, 2006).

[24] R. N. Barnett and K. B. Whaley, J. Chem. Phys. 99, 9730 (1993).

[25] D. Blume, M. Lewerenz, F. Huisken, and M. Kaloudis, J. Chem. Phys. 105, 8666 (1996).

[26] E. Lee, D. Farrelly, and K. B. Whaley, Phys. Rev. Lett. 83, 3812 (1999).

[27] Y. Kwon, D. M. Ceperley, and K. B. Whaley, J. Chem. Phys. 104, 2341 (1996).

[28] Y. Kwon, P. Huang, M. V. Patel, D. Blume, and K. B. Whaley, J. Chem. Phys. 113, 6469 (2000).

[29] F. Paesani, F. A. Gianturco, and K. B. Whaley, J. Chem. Phys. 115, 10225 (2001).

[30] S. Moroni, A. Sarsa, S. Fantoni, K. E. Schmidt, and S. Baroni, Phys. Rev. Lett. 90, 143401 (2003).

[31] J. Tang, A. R. W. McKellar, F. Mezzacapo, and S. Moroni, Phys. Rev. Lett. 92, 145503 (2004).

[32] F. Paesani and K. B. Whaley, J. Chem. Phys. 121, 5293 (2004).

[33] S. Moroni, N. Blinov, and P.-N. Roy, J. Chem. Phys. 121, 3577 (2004).

[34] R. E. Zillich, Y. Kwon, and K. B. Whaley, Phys. Rev. Lett. 93, 250401 (2004).

[35] R. E. Zillich and K. B. Whaley, Phys. Rev. B 69, 104517 (2004).

[36] F. Paesani, Y. Kwon, and K. B. Whaley, Phys. Rev. Lett. 94, 153401 (2005).

[37] R. E. Zillich, F. Paesani, Y. Kwon, and K. B. Whaley, J. Chem. Phys. 123, 114301 (2005).

[38] S. Paolini, S. Fantoni, S. Moroni, and S. Baroni, J. Chem. Phys. 123, 114306 (2005).

[39] W. Topic, W. Jäger, N. Blinov, P.-N. Roy, M. Botti, and S. Moroni, J. Chem. Phys. 125, 144310 (2006).

[40] A. Viel, K. B. Whaley, and R. J. Wheatley, J. Chem. Phys. 127, 194303 (2007).

[41] T. Škrbić, S. Moroni, and S. Baroni, J. Phys. Chem. A 111, 7640 (2007). 
[42] S. Miura, J. Chem. Phys. 126, 114308 (2007).

[43] K. von Haeften, S. Rudolph, I. Simanovski, M. Havenith, R. E. Zillich, and K. B. Whaley, Phys. Rev. B 73, 054502 (2006).

[44] R. E. Zillich and K. B. Whaley, J. Phys. Chem. A 111, 7489 (2007).

[45] N. D. Markovskiy and C. H. Mak, J. Phys. Chem. A 113, 9165 (2009).

[46] R. Rodríguez-Cantano, T. González-Lezana, and P. Villarreal, Int. Rev. Phys. Chem. 35, 37 (2016).

[47] R. Schmidt and M. Lemeshko, Phys. Rev. Lett. 114, 203001 (2015).

[48] R. Schmidt and M. Lemeshko, Phys. Rev. X 6, 011012 (2016).

[49] M. Lemeshko and R. Schmidt, in Low Energy and Low Temperature Molecular Scattering, edited by A. Osterwalder and O. Dulieu (RSC, Cambridge, 2017).

[50] B. Midya, M. Tomza, R. Schmidt, and M. Lemeshko, Phys. Rev. A 94, 041601(R) (2016).

[51] E. S. Redchenko and M. Lemeshko, ChemPhysChem 17, 3649 (2016).

[52] X. Li, R. Seiringer, and M. Lemeshko, arXiv:1610.04908.

[53] E. Yakaboylu and M. Lemeshko, arXiv:1612.02820.

[54] B. Shepperson, A. A. Søndergaard, L. Christiansen, J. Kaczmarczyk, R. E. Zillich, M. Lemeshko, and H. Stapelfeldt (to be published).

[55] D. A. Varshalovich, A. Moskalev, and V. Khersonskii, Quantum Theory of Angular Momentum (World Scientific, Singapore, 1988).

[56] C. H. Townes and A. L. Schawlow, Microwave Spectroscopy (Dover, New York, 1975).

[57] P. F. Bernath, Spectra of Atoms and Molecules, 2nd ed. (Oxford University Press, New York, 2005).

[58] A. Leggett, S. Chakravarty, A. T. Dorsey, M. P. A. Fisher, A. Garg, and W. Zwerger, Rev. Mod. Phys. 59, 1 (1987).

[59] R. Machleidt and D. Entem, Phys. Rep. 503, 1 (2011).

[60] E. Fradkin, Field Theories of Condensed Matter Physics, 2nd ed. (Cambridge University Press, Cambridge, England, 2013).

[61] R. Lehnig, P. L. Raston, and W. Jäger, Faraday Discuss. 142, 297 (2009).

[62] H. Katsuki and T. Momose, Phys. Rev. Lett. 84, 3286 (2000).

[63] A. Stone, The Theory of Intermolecular Forces (Oxford University Press, New York, 2013).

[64] L. Zang, W. Dai, L. Zheng, C. Duan, Y. Lu, and M. Yang, J. Chem. Phys. 140, 114310 (2014).

[65] A. Kalemos, Á. Valdés, and R. Prosmiti, J. Chem. Phys. 137, 034303 (2012).

[66] L. Garcia-Gutierrez, L. Delgado-Tellez, A. Valdes, R. Prosmiti, P. Villarreal, and G. Delgado-Barrio, J. Phys. Chem. A 113, 5754 (2009).

[67] K. A. Peterson and G. C. McBane, J. Chem. Phys. 123, 084314 (2005).

[68] J. M. M. Howson and J. M. Hutson, J. Chem. Phys. 115, 5059 (2001).

[69] B. T. Chang, O. Akin-Ojo, R. Bukowski, and K. Szalewicz, J. Chem. Phys. 119, 11654 (2003).

[70] H. Ran and D. Xie, J. Chem. Phys. 128, 124323 (2008).
[71] C. Callegari, I. Reinhard, K. K. Lehmann, G. Scoles, K. Nauta, and R. E. Miller, J. Chem. Phys. 113, 4636 (2000).

[72] G. Paterson, S. Marinakis, M. L. Costen, K. G. McKendrick, J. Kłos, and R. Toboła, J. Chem. Phys. 129, 074304 (2008).

[73] T. G. A. Heijmen, R. Moszynski, P. E. S. Wormer, and A. van der Avoird, J. Chem. Phys. 107, 9921 (1997).

[74] J. Kłos, G. Chałasiński, M. T. Berry, R. Bukowski, and S. M. Cybulski, J. Chem. Phys. 112, 2195 (2000).

[75] B. K. Taylor and R. J. Hinde, J. Chem. Phys. 111, 973 (1999).

[76] R. Moszynski, P. E. S. Wormer, B. Jeziorski, and A. van der Avoird, J. Chem. Phys. 101, 2811 (1994).

[77] G. Murdachaew, K. Szalewicz, H. Jiang, and Z. Bacic, J. Chem. Phys. 121, 11839 (2004).

[78] B. Fernandez, C. Henriksen, and D. Farrelly, Mol. Phys. 111, 1173 (2013).

[79] K. Harada, K. Tanaka, T. Tanaka, S. Nanbu, and M. Aoyagi, J. Chem. Phys. 117, 7041 (2002).

[80] P. J. Dagdigian and M. H. Alexander, J. Chem. Phys. 135, 064306 (2011).

[81] M. P. Hodges and R. J. Wheatley, J. Chem. Phys. 114, 8836 (2001).

[82] K. Patkowski, T. Korona, R. Moszynski, B. Jeziorski, and K. Szalewicz, J. Mol. Struct. 591, 231 (2002).

[83] R. T. Pack, E. Piper, G. A. Pfeffer, and J. P. Toennies, J. Chem. Phys. 80, 4940 (1984).

[84] G. Calderoni, F. Cargnoni, R. Martinazzo, and M. Raimondi, J. Chem. Phys. 121, 8261 (2004).

[85] See Supplemental Material at http://link.aps.org/ supplemental/10.1103/PhysRevLett.118.095301 for details.

[86] H. Lefebvre-Brion and R.W. Field, The Spectra and Dynamics of Diatomic Molecules (Elsevier, New York, 2004).

[87] L. C. Biedenharn and J. D. Louck, Angular Momentum in Quantum Physics (Addison-Wesley, Reading, MA, 1981).

[88] R. E. Zillich (private communication).

[89] O. Akin-Ojo, R. Bukowski, and K. Szalewicz, J. Chem. Phys. 119, 8379 (2003).

[90] K. Nauta and R. E. Miller, J. Chem. Phys. 115, 10254 (2001).

[91] A. Conjusteau, C. Callegari, I. Reinhard, K. K. Lehmann, and G. Scoles, J. Chem. Phys. 113, 4840 (2000).

[92] K. Nauta and R. E. Miller, J. Chem. Phys. 115, 8384 (2001).

[93] K. von Haeften, A. Metzelthin, S. Rudolph, V. Staemmler, and M. Havenith, Phys. Rev. Lett. 95, 215301 (2005).

[94] D. Skvortsov, M. Y. Choi, and A. F. Vilesov, J. Phys. Chem. A 111, 12711 (2007).

[95] K. Nauta and R. E. Miller, J. Chem. Phys. 113, 9466 (2000).

[96] P. L. Raston, T. Liang, and G. E. Douberly, J. Phys. Chem. A 117, 8103 (2013).

[97] M. N. Slipchenko and A. F. Vilesov, Chem. Phys. Lett. 412, 176 (2005).

[98] A. M. Morrison, P. L. Raston, and G. E. Douberly, J. Phys. Chem. A 117, 11640 (2013).

[99] K. E. Kuyanov, M. N. Slipchenko, and A. F. Vilesov, Chem. Phys. Lett. 427, 5 (2006). 
[100] M. Hartmann, R. E. Miller, J. P. Toennies, and A. Vilesov, Phys. Rev. Lett. 75, 1566 (1995).

[101] K. Nauta and R. E. Miller, Chem. Phys. Lett. 350, 225 (2001).

[102] M. O. Scully and M.S. Zubairy, Quantum Optics (Cambridge University Press, Cambridge, England, 1997).

[103] T. Rentrop, A. Trautmann, F. A. Olivares, F. Jendrzejewski, A. Komnik, and M. K. Oberthaler, Phys. Rev. X 6, 041041 (2016).
[104] D. Pentlehner, J. H. Nielsen, A. Slenczka, K. Mølmer, and H. Stapelfeldt, Phys. Rev. Lett. 110, 093002 (2013).

[105] D. Pentlehner, J. H. Nielsen, L. Christiansen, A. Slenczka, and H. Stapelfeldt, Phys. Rev. A 87, 063401 (2013).

[106] L. Christiansen, J. H. Nielsen, D. Tobias, V. Pentlehner, J. G. Underwood, and H. Stapelfeldt, Phys. Rev. A 92, 053415 (2015). 